\title{
Analysis of microstrip antennas with finite-sized substrate
}

Yi-Hsin Pang* and Ruey-Beei Wu

Department of Electrical Engineering

National Taiwan University, Taipei, Taiwan, R.O.C.

E-mail: d85014@ew.ee.ntu.edu.tw, rbwu@ew.ee.ntu.edu.tw

Abstract - Effects of the finite-sized substrate and ground plane on the radiation characteristics of a microstrip antenna are studied. Applying the boundary integral method, the electric and magnetic fields radiation on the dielectric boundary of the finite microstrip structures can be solved numerically, without the complicated Sommerfeld integral and the assumption of infinite substrate. Rectangular microstrip antennas with finite-sized substrate and ground plane are analyzed, and the numerical results are compared to the experimental data.

\section{INTRODUCTION}

Microstrip antennas are widely used, since they are lightweight, conformal and easy to fabricate. Analyses of microstrip antennas are also available in extensive literature. Integral equation utilized Sommerfeld formulation is one of the popular full-wave analysis methods, such as [1]. An important assumption of this method is that the substrate and ground plane are of infinite extent in the transverse direction. Without this assumption, the associated Green's functions are not available and the method fails. This assumption is not practical, however, especially for the application of personal wireless communications. The diffraction due to the presence of the edges of substrate and ground plane would affect the various characteristics of antennas, such as the resonant frequency[2], input impedance[3], and radiation patterns[4]. Several methods have been proposed in the literature to deal with such problems[5]-[7]. In this study, we adopt the surface/surface formulation proposed in [5] with more rigorous integration method, and the results of input impedance that are not presented in [5] are given.

\section{IJ. OUTLINE OF THE THEORY}

Fig. 1(a) depicts the problem of interest. Denote the boundary of the whole antenna structure as surface $S$, inside which a linear, isotropic, and homogeneous substrate with relative permittivity $\varepsilon_{\mathrm{r}}$ and relative permeability $\mu_{\mathrm{r}}$ is assumed. $\mathbf{J}^{\mathbf{i}}$ and

This work was supported in part by the National Science Council, Republic of China, under Grant NSC 88-2213-E-002-074, and the Ministry of Education under Grant 89-E-FA06-2-4. 
$\mathbf{M}^{\mathbf{i}}$ represent the feeding sources for the antenna. Outside $\mathrm{S}$ is free space where the antenna radiates. Another assumption imposed is that metals are of zero thickness and, although not necessary, are perfectly electrical conductors (PEC). By applying the equivalence principle on the surface $\mathrm{S}$, the original problem is divided into two equivalent problems[8], as shown in Fig. 1(b) and Fig. 1(c). The fields in both of the problems can be expressed in terms of the feeding source $\left(\mathbf{J}^{\mathbf{l}}, \mathbf{M}^{\mathbf{l}}\right)$ and the equivalent surface current $\left(\mathbf{J}_{s}, \mathbf{M}_{s}\right)$ on the dielectric boundary. Note that the equivalent surface currents are not independent at all for the exterior and interior problems. Because of the continuity of electric field and magnetic field, we must have $\mathbf{M}_{\text {s,int }}=-\mathbf{M}_{\mathrm{s}, \mathrm{ext}}$ and $J_{s, i n t}=-J_{s, \text { xx }}$ on the dielectric portion of S. Matching the boundary conditions on $S$, we can obtain coupled integral equations for the unknown equivalent surface currents.

With the method of moments, the integral equations can be cast into a matrix form and solved numerically by LU decomposition. The same basis and weighting functions as those indicated in [5] are chosen, but more rigorous integration is performed.

\section{RESULTS}

Take the rectangular microstrip antenna shown in Fig. 2 as the first example. At first, we examine the formulations when the relative dielectric constant is 1.0 , i.e. the slab does not actually exist. This antenna was respectively solved by integral equation method with free space Green's function and by this method where the slab exists and $\varepsilon_{r}$ of the slab is set to 1,0 . Good agreement that is not shown here is obtained for frequency ranging from $0.1 \mathrm{GHz}$ to $9.0 \mathrm{GHz}$.

Chen[9] analyzed the above antenna by integral equation method under the assumption of infinite substrate with relative dielectric constant 10.2 and loss tangent 0.001 . In this study, the dielectric constant is set to 10.0 and the substrate is lossless and finite. A voltage gap source is used for excitation. The numerical result is shown in Fig. 2 and is consistent with [9]

A microstrip antenna fabricated on the FR4 substrate of finite extent is used to verify the numerical analysis. The transverse dimension of the substrate varies from 6 $\mathrm{cm} \times 5 \mathrm{~cm}$ to $4 \mathrm{~cm} \times 3 \mathrm{~cm}$, as shown in Fig. 3. In the numerical analysis, the relative dielectric constant is set to 4.55 . The measured data and the numerical results shown in Fig. 3 do not coincide very well. The numerical results have smaller bandwidth mostly because of the assumption of lossless substrate. However, as can be observed in Fig. 3, both results show that the resonant frequency becomes smaller as the size of the slab decreases 


\section{SUMMARY}

In this paper, microstrip antennas with substrate of finite extent are analyzed by the boundary integral method. Numerical data are also compared with the experimental results. It shows that the boundary integral method works for the analysis of microstrip antennas with finite-sized substrate. It can also be applied to discuss the finite dielectric effects on the radiation characteristics of other printed antennas such as the CPW-fed microstrip antennas. Besides, extension of the present method to deal with structures of lossy and inhomogeneous substrate deserves further study.

\section{REFERENCE}

[1] J. R. Mosig, "Arbitrarily shaped microstrip structures and their analysis with a mixed potential integral equation," IEEE Trans. Microwave Theory Tech., vol. 36, pp. 314-323. Feb. 1988.

[2] M. Gimersky and J. Bornemann, "A modified method-of-moments technique for the full-wave analysis of imperfect conductors on lossy and finite-extent substrates," IEEE MTT-S Digest, pp. 715-718, 1996.

[3] H. Iwasaki, "A back-to-back rectangular-patch antenna fed by a CPW," IEEE Trans. Antennas Propagat., vol. 46, no. 10, pp. 1527-1530, Oct. 1998.

[4] J. Huang, "The finite ground plane effect on the microstrip antenna radiation patterns," IEEE Trans. Antennas Propagat., vol. 31, no. 4, pp. 649-653, July 1983.

[5] T. K. Sarkar, S. M. Rao, and A. R. Djordjević, "Electromagnetic scattering and radiation T. K. Sarkar, S. M. Rao, and A. R. Djordjevic, "Electromagnetic scattering and radiation
from finite microstrip structures," IEEE Trans. Microwave Theory Tech, vol. 38, no. 11,

[6] S. A. Bokhari, J. R. Mosig, and F. E. Gardiol, "Radiation pattern computation of microstrip antennas on finite size ground planes," IEE Proc.-H, vol. 139, No. 3, pp. 278-286, June 1992.

[7] M. Drissi, P. Herve, and J. Citerne, "A rigorous analysis of planar antennas having a finite size substrate," IEEE Int. Symp. Dig. Antennas Propagat, pp. 1462-1465, 2000.

[8] R. F. Harrington, Time-harmonic electromagnetic fields, McGraw-Hill, 1993.

[9] W. Chen, K. F. Lee, and R. Q. Lee, "Input impedance of coaxialiy fed rectangula microstrip antenna on electrically thick substrate," Microwave Opt. Technol. Lett., vol. 6, no. 6, pp. 387-390, 1993.

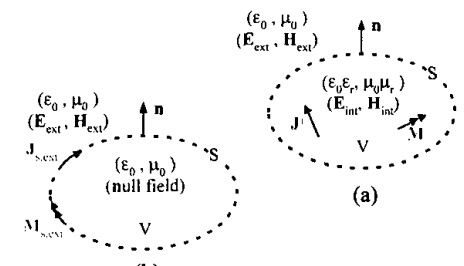

(b)

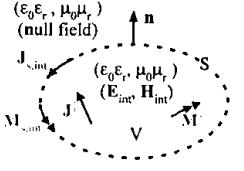

(c)

Fig. 1. Problem of interest; (a) the original problem, (b) the exterior equivalent problem, and (c) the interior equivalent problem. 


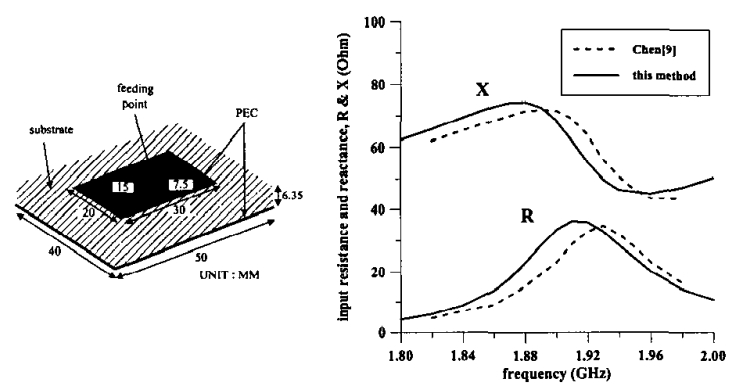

Fig. 2. Input impedance of a rectangular microstrip antenna with finite-sized substrate and ground plane. In the result of Chen, $\varepsilon_{\mathrm{r}}=10.2$ and $\tan \delta=0.001$ for the infinite substrate. In this method, $\varepsilon_{\mathrm{r}}=10$ and lossless for the finite-sized slab.

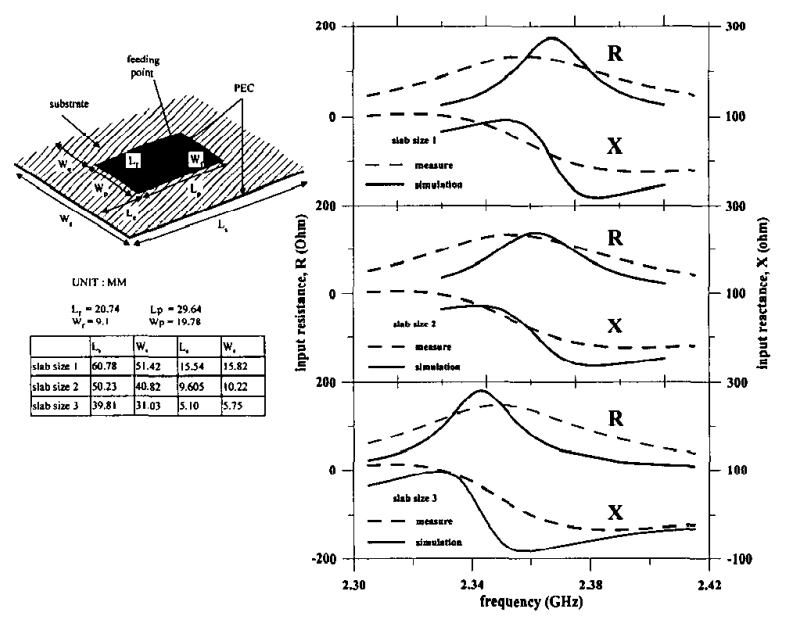

Fig. 3. Numerical analysis of a microstrip antenna with finite-sized substrate (FR4) and ground plane, compared with the experimental results. 